\title{
Cocoa polyphenols treatment ameliorates visceral obesity by reduction lipogenesis and promoting fatty acid oxidation genes in obese rats through interfering with AMPK pathway
}

\begin{abstract}
This study was conducted to investigate the pharmacological activity of cocoa polyphenols (CPs) on visceral obesity markers and the possible mechanisms. In this study, Spragueï Dawley (SD) rats were fed either a low-fat diet (LFD) or a high-fat diet (HFD). After 12 $\mathbb{k} k \mathrm{k}$

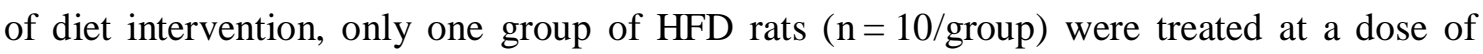

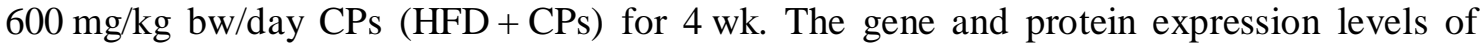
phosphorylation of AMPK-activated protein kinase Ǔ/Б (AMPK Ǔðб) were measured using real time-PCR and Western blotting. The mRNA expression of lipogenic key enzymes (Acaca, Fasn, Mcat, and Scd-1), and 6-oxidation key enzymes (CPT1, Prkaa1, Acox1) were investigated. In addition, the upstream transcription factors (PPARŬ, PPARJ, C/EBPŬ, and SREBP-1c) were also examined. In accordance with these findings, CPs treatment improved visceral adiposity, adipocytes hypertrophy, and liver steatosis. AMP-activated protein kinase

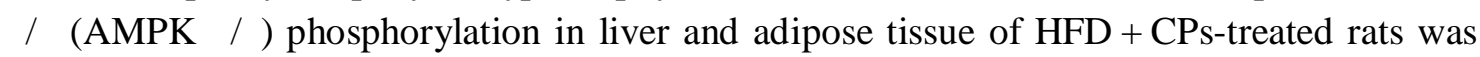
activated compared with HFD-fed rats. The expression of lipogensis related-genes was decreased, while expression levels of b-oxidation-related genes were increased compared with HFD-fed rats. Together, these data partially unravel the ameliorative effects of CPs treatment on visceral obesity markers by inhibiting lipogenesis and promoting b-oxidation related-genes through activation of the AMPK pathway.
\end{abstract}

Keyword: AMPK pathway; Cocoa polyphenols; Fatty acid 6-oxidation genes; Lipogenesis genes; Visceral obesity 